J. Dairy Sci. 91:4115-4115

doi:10.3168/jds.2008-1644

(c) American Dairy Science Association, 2008.

\title{
Letter to the Editor: A Response to the Comments of Bendall and Pearce (2008)
}

\author{
M. W. Schilling ${ }^{1}$ \\ Department of Food Science, Nutrition, and Health Promotion, Mississippi State University, Mississippi State 39762
}

The authors of the letter to the editor (Bendall and Pearce, 2008) that was written regarding our recent paper (Gandy et al., 2008) are correct to bring up some concerns regarding interpretations that could be made in respect to volatile composition and product safety. In addition, we did not intend to infer that milk pasteurization (at any temperature/time combination) is unsafe. The true intent of our paper was to determine if altering the pasteurization temperature (because dairy plants vary in their pasteurization temperature and have been steadily increasing pasteurization temperature over the past few years in the United States) affects milk quality (consumer and sensory) and shelflife. The references to Mycobacterium tuberculosis were correctly stated but did not include all available references on the subject. It was therefore unnecessary for us to include these references in the paper because they could be misleading to the journal's audience as we definitely consider pasteurization of milk to be a safe process.

As the corresponding author, I am confident that all of the results of this research are sound with the exception of some of the volatile composition data. Bendall and Pearce (2008) are correct in stating that GC-MS spectra are difficult to interpret and that the only data that are definitively correct are the $11 \mathrm{com}$ pounds verified with authentic standard compounds. The other compounds were tentatively identified, and it is correct (as stated in the letter to the editor) that ethylamine, guanidine, hydroxylamine, hydrogen peroxide, and tetrahydrofuran should not have been listed in the data because it is not reasonable that these compounds would be in the milk product and verification with authentic standards was not performed. It is with my sincere apologies that I missed this error and that these compounds were included in the text. The remainder of the volatile compounds that were tentatively identified were also not verified with authentic standards, but it is reasonable that these compounds could be in the milk and that they could be reported as tentatively identified (a common, albeit debatable, practice in food science journals). It is also correct, as stated by Bendall and Pearce (2008), that for a chemistry journal, authentic standards are required and should be utilized to ensure the identification of volatile compounds. However, it would also be a good argument for this to be the policy for all scientific journals, including food science journals. In addition, standard blank runs (with solid-phase microextraction fibers) were always run in the GC-MS before analysis of milk samples in the study.

It is well known that volatile compounds may vary between milk samples, and the same base milk was utilized for each replication of this study. In addition, subsamples were utilized within each replication for all volatile component analyses. Once again, I apologize for the inclusion of ethylamine, guanidine, hydroxylamine, hydrogen peroxide, and tetrahydrofuran in the GC-MS data results. We want to thank Bendall and Pearce (2008) for maintaining the scientific integrity of our community by pointing out this mistake and ensuring that readers would not mistakenly think that milk pasteurization is an unsafe process.

\section{REFERENCES}

Bendall, J. G., and L. E. Pearce. 2008. Letter to the Editor: Pasteurization temperature of milk: A comment on Gandy et al. (2008). J. Dairy Sci. 91:4113-4114.

Gandy, A. L., M. W. Schilling, P. C. Coggins, C. H. White, Y. Yoon, and V. V. Kamadia. 2008. The effect of pasteurization temperature on consumer acceptability, sensory characteristics, volatile compound composition, and shelf-life of fluid milk. J. Dairy Sci. 91:1769-1777.

Received August 20, 2008.

Accepted September 15, 2008

${ }^{1}$ Corresponding author: schilling@foodscience.msstate.edu 